\title{
Effect of maternal clinical chorioamnionitis on neonatal morbidity in very-low birthweight infants: a case-control study
}

Francesc Botet $^{1, *}$, Josep Figueras ${ }^{1}$, Xavier CarbonellEstrany $^{1}$, Gemma Arca ${ }^{1}$ and the Castrillo Study Group $^{2}$

${ }^{1}$ Neonatology Service, Hospital Clínic de Barcelona, Universitat de Barcelona, Barcelona, Spain

${ }^{2}$ Castrillo Study Group: M. José Párraga Quiles and Juana M. Guzmán, Hospital Reina Sofía, Córdoba; Ana Alarcón Allen, Hospital Sant Joan de Déu, Esplugues de Llobregat, Barcelona; Carmen González Armengod, Hospital Clínico, Valladolid; Ana Remesal Escalero, Hospital Universitario de Salamanca, Salamanca; María J. García García, Hospital San Pedro de Alcántara, Complejo Hospitalario de Cáceres, Cáceres; Emilio Álvaro Iglesias, Hospital de León, León; Roser Porta, Institut Universitari Dexeus, Barcelona; Rafael Reparaz, Complejo Hospitalario Juan Canalejo, A Coruña; Eduardo Narbona, Hospital Clínico, Granada; Belén Colomer, Hospital Central de Asturias, Oviedo; and José Martínez Orgado, Fundación Hospital Alcorcón, Madrid, Spain

\footnotetext{
Abstract birthweight (VLBW) infants. case.

* Corresponding author:

Francesc Botet, MD, PhD

Neonatology Service

Hospital Clínic de Barcelona

Sabino de Arana 1

E-08028 Barcelona

Spain

Tel.: + 34-93-2275600

Fax: +34-93-2275605

E-mail: fbotet@clinic.ub.es
}

Aims: To assess the relationship between maternal clinical chorioamnionitis and neonatal outcome in preterm very-low

Methods: An observational case-control study was conducted in the Neonatology Services of 12 acute-care teaching hospitals in Spain. Between January 2004 and December 2006, all consecutive VLBW ( $\leq 1500 \mathrm{~g}$ ) infants born to a mother with clinical chorioamnionitis were enrolled. Controls were infants without chorioamnionitis matched by gestational age who were born immediately after each index

Results: There were 165 cases and 163 controls. A significantly higher percentage of cases than controls required intubation (53\% vs. $35.8 \%$ ), had normal intrauterine growth ( $98.1 \%$ vs. $84.7 \%$ ), were born in a tertiary center (inborn) ( $95.1 \%$ vs. $89.1 \%$ ), from single gestations $(76.4 \%$ vs. $65.6 \%)$ and vaginal delivery $(47.3 \%$ vs. $33.3 \%)$, showed a lower
Apgar score at $5 \mathrm{~min}$, and presented a higher rate of earlyonset sepsis (10.4\% vs. $1.2 \%)$. Older maternal age (32.5 vs. 30.8 years), premature labor $(67.3 \%$ vs. $25.8 \%)$, premature rupture of membranes $(61.3 \%$ vs. $25.8 \%)$, and antibiotic treatment $(88.5 \%$ vs. $52.3 \%)$ were significantly more frequent among cases than controls.

Conclusions: After controlling by gestational age, maternal chorioamnionitis was associated with neonatal depression and early sepsis but not with other prematurity-related complications.

Keywords: Chorioamnionitis; fetal membranes; infant, premature; premature birth; premature rupture.

\section{Introduction}

Chorioamnionitis is considered to be one of the main causes of preterm delivery and has been associated with adverse perinatal outcomes in preterm infants. Preterm delivery remains a major obstetric problem, accounting for $70 \%$ of perinatal mortality and nearly $50 \%$ of long-term neurologic morbidity [5]. In recent years, a great deal of attention has turned to the impact of antenatal inflammation on both shortterm outcome and long-term sequelae in preterm infants. In addition to severe acute morbidity, including early-onset sepsis, necrotizing enterocolitis and lung disease [2, 14], the preterm infant is at considerable increased risk of developing intraventricular hemorrhage and periventricular leukomalacia, which, in turn, is a strong predictor of mental retardation and cerebral palsy $[8,22,23]$. Histological chorioamnionitis is associated with a decreased incidence of respiratory distress syndrome, whereas the incidence of bronchopulmonary dysplasia is increased [1]. On the other hand, full-term babies exposed to intrauterine infection often present with depressed Apgar scores and neonatal encephalopathy, and are at marked increased risk of developing cerebral palsy.

On the basis of the close association between infection and very preterm birth, some authors have proposed that chorioamnionitis plays a causal role not only in the induction of delivery, but also in producing the fetal inflammatory response syndrome, which is also considered as the cause of acute morbidity as well as long-term sequelae in the infant $[8,18]$. Different studies have examined the effects of clinical maternal chorioamnionitis and histological chorioamnionitis on neonatal and neurodevelopmental outcome in preterm babies [13, 19-21]. However, the interpretation of these studies is complicated by the fact that important confounders, such as gestational age have not been adjusted for 
in the analysis. Therefore, a case-control study in which cases and controls were matched by gestational age was designed to assess the relationship between maternal clinical chorioamnionitis and neonatal outcome in very-low birthweight (VLBW) preterm infants.

\section{Methods}

Between January 2004 and December 2006, the Neonatology Services of 12 acute-care university-affiliated hospitals throughout Spain participated in an observational, prospective, case-control study. Cases were all consecutive preterm infants weighing $\leq 1500 \mathrm{~g}$ who were born to a mother with history of clinical chorioamnionitis. Controls were infants without such history matched by gestational age, who were born subsequently to each index case. The diagnosis of clinical chorioamnionitis in the pregnant woman who presented with premature labor or premature rupture of the membranes was made in the presence of uterine pain, fever $\left(\geq 38^{\circ} \mathrm{C}\right)$, leukocytosis $\left(>12,000\right.$ cells $\left./ \mathrm{mm}^{3}\right)$, and increased serum concentrations of Creactive protein $(>30 \mathrm{mg} / \mathrm{L})$. Common clinical manifestations, such as malodorous vaginal discharge and maternal or fetal tachycardia were not included as diagnostic criteria of clinical chorioamnionitis in the study protocol. Infants with chromosomal or congenital abnormalities were excluded from the study. The study was approved by the Institutional Review Board of each participating center, and written informed consent from the parents for data collection was obtained. The inclusion of cases and controls in the study did not involve any intervention. Infants received individualized standard care according to current clinical guidelines.

A study protocol was designed in which the following data were prospectively recorded: maternal age; premature labor (persistent uterine contraction, morphological changes in the cervix); premature rupture of membranes (clinical or biochemical evidence of amniorrhexis); intrapartum antibiotic treatment; antenatal steroid therapy (one or more course of betamethasone); cesarean delivery; multiple gestation; and whether the birth occurred in or outside the tertiary care center (inborn, outborn). Preterm labor was usually defined as regular contractions accompanied by cervical changes at $<37$ weeks' gestation [4]. Morphological changes in the cervix included $2 \mathrm{~cm}$ dilatation or short cervical length $(<25 \mathrm{~mm})$ [9]. Premature rupture of membranes was defined as spontaneous rupture of membranes at $<37$ weeks' gestation at least $1 \mathrm{~h}$ before the onset of contractions. Diagnosis can be made clinically by evidence of amniotic fluid in the vagina ( $\mathrm{pH}$ testing, fluorescein injection) or biochemically (fetal fibronectin testing) [3]. Newborn data included gestational age, sex, weight at birth, intrauterine growth restriction (IUGR) (below the $10^{\text {th }}$ percentile using race- and sex-specific growth charts); need of resuscitation maneuvers in the delivery room using endotracheal intubation, and Apgar score at $5 \mathrm{~min}$. Neonatal morbidity and mortality data included the presence of respiratory distress syndrome, patent ductus arteriosus (and surgical correction), necrotizing enterocolitis (and surgical treatment), seizures, early-onset sepsis, late-onset sepsis, fungal sepsis, intraventricular hemorrhage (and severe intraventricular hemorrhage grades III and IV), periventricular leukomalacia, bronchopulmonary dysplasia or oxygen requirement at 28 days, chronic lung disease or oxygen requirement at a corrected age of 36 weeks gestation, retinopathy of prematurity (grade $\geq 2$ ) (and laser treatment), death, and survival without chronic lung disease. The length of hospital stay was also recorded.
The Kolmogorov-Smirnov test was used to evaluate the distribution of data. Normally distributed data are expressed as mean and standard deviation (SD) and otherwise as median and interquartile $\left(25^{\text {th }}-75^{\text {th }}\right.$ percentile) range. Categorical variables are expressed as absolute numbers and percentages. Differences between cases and controls were assessed with the Mann-Whitney $U$-test or one-way analysis of variance (ANOVA) for continuous variables, and the chisquare $\left(\chi^{2}\right)$ test for categorical variables. Odds ratio and $95 \%$ confidence intervals (CI) were calculated to assess the magnitude of the differences. The SPSS statistical package was used (version 12.0, SPSS Inc., Chicago, IL). P-value of $<0.05$ was considered statistically significant.

\section{Results}

A total of 328 preterm babies weighing $\leq 1500 \mathrm{~g}$ were recruited during the study period, and grouped into 165 cases and 163 controls. Perinatal characteristics are shown in Table 1. Cases and controls were similar regarding weight at birth, gestational age, and sex. IUGR occurred less frequently in the group of infants with maternal chorioamnionitis than in controls. In this group there were also more inborns, singletons, and vaginal deliveries. Moreover, resuscitation with endotracheal intubation was needed more frequently and had a lower 5-min Apgar score. Premature labor, premature rupture of membranes, and antimicrobial therapy were also more frequent among cases than controls.

As shown in Table 2, morbidity and mortality was similar in both study groups. The only variable with significant differences was early-onset sepsis, which occurred in $10.4 \%$ of infants with maternal chorioamnionitis and in $1.2 \%$ in controls $(\mathrm{P}=0.001)$ (odds ratio 9.3, 95\% CI 2.11-9.31). The mortality rate was $23 \%$ in cases and $17.2 \%$ in controls. The median length of hospital stay was 56 days in both cases and controls.

\section{Discussion}

In the present prospective case-control study of VLWB preterm infants, those born from mothers with clinical chorioamnionitis were more depressed at birth, required more often neonatal resuscitation and endotracheal intubation, and suffered from early-onset sepsis more frequently than controls. These findings are consistent with a previous casecontrol study carried out by our group on 135 newborns weighing $<1500 \mathrm{~g}$ at birth and born between 1988 and 1998 [7]. In this study, the cases comprised of 45 newborns exposed to clinical or subclinical levels of maternal chorioamnionitis. Each newborn in the case group was matched with two controls, both weighing $<1500 \mathrm{~g}$, one of them born immediately before and the other immediately after. Perinatal records, neonatal morbidity and mortality were analyzed. Forty percent of the cases presented sepsis in the first $72 \mathrm{~h}$ of life compared with $10 \%$ of the controls $(\mathrm{P}<0.0001)$. In addition, resuscitation $(77.8 \%$ vs. $45.6 \%, \mathrm{P}=0.001)$ and mechanical ventilation $(73 \%$ vs. $50 \%, \mathrm{P}=0.016)$ were required by more cases than controls. The presence of cho- 
Table 1 Perinatal characteristics of the study population.

\begin{tabular}{|c|c|c|c|c|}
\hline Variables & $\begin{array}{l}\text { Cases } \\
\mathrm{n}=165\end{array}$ & $\begin{array}{l}\text { Controls } \\
\mathrm{n}=163\end{array}$ & P-value & $\begin{array}{l}\text { Odds ratio } \\
(95 \% \text { CI })\end{array}$ \\
\hline \multicolumn{5}{|l|}{ Maternal and labor data } \\
\hline Maternal age, years, mean (SD) & $32.5(5.3)$ & $30.8(5.5)$ & 0.006 & \\
\hline Premature labor & $111(67.3)$ & $92(56.4)$ & 0.043 & $1.59(1.01-2.48)$ \\
\hline Premature rupture of membranes & $98(59.4)$ & $41(25.1)$ & $<0.001$ & $4.55(2.82-7.33)$ \\
\hline Antibiotic treatment & $112(67.9)$ & $46(28.2)$ & $<0.001$ & $20.45(7.61-54.9)$ \\
\hline Antenatal steroids & $146(88.5)$ & $130(79.7)$ & 0.433 & \\
\hline Cesarean delivery & $87(52.7)$ & $108(66.2)$ & 0.010 & $0.56(0.36-0.88)$ \\
\hline Multiple gestation & $39(23.6)$ & $56(34.4)$ & 0.032 & $0.59(0.36-0.96)$ \\
\hline Inborn & $156(94.5)$ & $145(88.9)$ & 0.039 & $2.42(1.02-5.75)$ \\
\hline \multicolumn{5}{|l|}{ Newborn data } \\
\hline Males & $86(52.1)$ & $91(55.8)$ & 0.501 & \\
\hline Gestational age, weeks, median (IQR) & $28.0(26.4-29.7)$ & $28.7(27-29.8)$ & 0.107 & \\
\hline Birthweight, g, mean (SD) & $1073(246)$ & $1040(229)$ & 0.216 & \\
\hline IUGR & $13(7.9)$ & $25(15.3)$ & 0.035 & $0.47(0.23-0.96)$ \\
\hline Apgar score at 5 min, median (IQR) & $8(7-9)$ & $9(8-9)$ & $<0.001$ & \\
\hline Endotracheal intubation & $87(52.7)$ & $58(35.6)$ & 0.002 & $2.03(1.30-3.16)$ \\
\hline
\end{tabular}

Data as number and percentages unless otherwise stated.

$\mathrm{CI}=$ confidence interval, $\mathrm{SD}=$ standard deviation, $\mathrm{IUGR}=$ intrauterine growth restriction, $\mathrm{IQR}=$ interquartile range $\left(25^{\text {th }}-75^{\text {th }}\right.$ percentile $)$.

rioamnionitis was associated with a higher risk of early onset infection and the need for neonatal resuscitation and mechanical ventilation.

Chorioamnionitis has been also associated with premature rupture of membranes. In a review of 430 singleton pregnancies with confirmed premature preterm rupture of membranes at 24 weeks or beyond that resulted in delivery at $<37$ weeks, composite neonatal major and minor morbidity rates were compared between pregnancies complicated by chorioamnionitis and those that were not [16]. Thirteen per- cent of women with premature preterm rupture of membranes developed chorioamnionitis. The composite neonatal major morbidity rate was significantly higher in neonates whose mothers developed chorioamnionitis (55\%) vs. those who did not $(18 \%, \mathrm{P}<0.0001)$, and the incidence of chorioamnionitis increased significantly with decreasing gestational age. For this reason, in our study, cases and controls were matched by gestational age.

In a retrospective study on 452 preterm newborns $<34$ weeks at birth (mean birthweight $1440 \mathrm{~g}$ ) that evaluated

Table 2 Morbidity and mortality during the neonatal period.

\begin{tabular}{llll}
\hline Variables & $\begin{array}{l}\text { Cases } \\
\mathrm{n}=165\end{array}$ & $\begin{array}{l}\text { Controls } \\
\mathrm{n}=163\end{array}$ \\
\hline Respiratory distress syndrome & $101(61.2)$ & $109(66.9)$ & P-value \\
Patent ductus arteriosus & $52(31.5)$ & $60(36.8)$ & 0.391 \\
$\quad$ Surgical correction & $13 / 52(25)$ & $8 / 60(13.3)$ & 0.370 \\
Necrotizing enterocolitis & $15(9.1)$ & $9 / 14(64.3)$ & 0.114 \\
$\quad$ Surgical treatment & $8 / 15(53.3)$ & $5(3.1)$ & 0.888 \\
Seizures & $7(4.2)$ & $2(1.2)$ & 0.549 \\
Early-onset sepsis & $17(10.3)$ & $52(31.9)$ \\
Late-onset sepsis & $59(35.7)$ & $8(4.9)$ \\
Fungal sepsis & $17(10.3)$ & $45(27.6)$ \\
Intraventricular hemorrhage & $44(26.7)$ & $21(12.9)$ \\
$\quad$ IVH grade III-IV & $17(10.3)$ & $21(12.9)$ \\
Periventricular leukomalacia & $26(15.7)$ & $49(30.1)$ \\
Bronchopulmonary dysplasia & $49(29.7)$ & $27(16.6)$ \\
Chronic lung disease & $18(10.9)$ & $14(8.6)$ & 0.554 \\
Retinopathy of prematurity (grade $\geq 2)$ & $18(10.9)$ & $6 / 14(42.8)$ \\
$\quad$ Laser treatment & $6 / 18(33.3)$ & $28(17.2)$ \\
Death & $38(23.0)$ & $56(39.5-73)$ & 0.001 \\
Length of hospital stay, days, median (IQR) & $56(28-76)$ & $106(65.0)$ \\
Alive without chronic lung disease & $106(64.2)$ & 0.066 \\
\hline
\end{tabular}

Data as number and percentages unless otherwise stated.

$\mathrm{IQR}=$ interquartile range $\left(25^{\text {th }}-75^{\text {th }}\right.$ percentile $)$. 
the role of histological chorioamnionitis on the overall morbidity of preterm newborns, an association only between histological chorioamnionitis and cystic periventricular leukomalacia was observed [17]. In our study, examination of the placenta to confirm histological chorioamnionitis was not performed. However, the strength of association between histologic chorioamnionitis and preterm delivery has been inconsistent [10]. In another retrospective study of 100 women with singleton pregnancies between 22 and 28 weeks of gestation and with chorioamnionitis diagnosed on admission by amniotic fluid neutrophil elastase level, higher levels of this biochemical marker were associated with a shorter interval from admission to delivery [12]. In a retrospective comparison of adverse neonatal outcome of singleton pregnancies with documented premature rupture of membranes and chorioamnionitis (cases) with those who did not (controls), poor neonatal outcome was significantly associated with chorioamnionitis ( $34 \%$ vs. $13 \%, \mathrm{P}=0.008)$, confirming that chorioamnionitis complicating premature rupture of membranes worsens neonatal outcome [15].

In contrast with studies with a retrospective cohort design, the present prospective case-control study suggests that after controlling for gestational age, complications, especially related to prematurity rather than to chorioamnionitis, did not appear to be significant. An increase in the occurrence of intraventricular hemorrhage, severe intraventricular hemorrhage, periventricular leukomalacia, bronchopulmonary dysplasia, and chronic lung disease in the chorioamnionitis group was not observed. According to our findings, maternal chorioamnionitis was a risk factor for neonatal depression and early-onset sepsis.

Inflammation has been implicated in the mechanisms responsible for preterm and term parturition, as well as fetal injury. Most cases of histopathological inflammation and histological chorioamnionitis, both in preterm and term labors, are subclinical in nature, and the isolation of bacteria in the amniotic fluid is a pathological finding, the frequency of which is dependent upon the clinical presentation and gestational age [18]. The natural history of inflammation after premature rupture of membranes can be modified by antibiotic administration, although antibiotic administration rarely eradicates intra-amniotic infection in patients with preterm premature rupture of membranes; moreover, some patients with documented inflammation of the amniotic cavity show a decrease in the intensity of the inflammatory process after antibiotic administration [6]. Antenatal inflammation is very commonly associated with preterm deliveries, but there is generally minimal information about the duration, intensity, or organisms associated with chorioamnionitis. In preterm animal models, chorioamnionitis causes a lung injury similar to bronchopulmonary dysplasia and also causes clinical lung maturation. Continuous exposure of the developing lung before and after delivery to inflammation may be central to the development of bronchopulmonary dysplasia [11]. It might be plausible that antimicrobials and antenatal steroids in pregnant women with chorioamnionitis may decrease the inflammatory response. In the present series, $88.5 \%$ of women received antenatal steroids, which may account for the favorable outcome of neonates in the chorioamnionitis group. However, the infectious/inflammatory mechanisms involved in acute neonatal morbidity and mortality in preterm infants exposed to chorioamnionitis are incompletely understood.

Although in this prospective clinical study the diagnosis of histological chorioamnionitis was not made, we conclude that after controlling by gestational age, maternal clinical chorioamnionitis was associated with neonatal depression and early-onset sepsis but not with other prematurity-related complications.

\section{Acknowledgment}

We thank Marta Pulido, MD, for editing the manuscript and editorial assistance.

\section{References}

[1] Been JV, Zimmermann LJI. Histological chorioamnionitis and respiratory outcome in preterm infants. Arch Dis Child Fetal Neonatal Ed. 2009;94:F218-25.

[2] Bracci R, Buonocore G. Chorioamnionitis: a risk factor for fetal and neonatal morbidity. Biol Neonate. 2003;83:85-96.

[3] Faye-Petersen OM. The placenta in preterm birth. J Clin Pathol. 2008;61:1261-75.

[4] Goldenberg RL, Culhane JF, Iams JD, Romero R. Epidemiology and causes of preterm birth. Lancet. 2008;371:75-84.

[5] Goldenberg RL, Hauth JC, Andrews WW. Intrauterine infection and preterm delivery. N Engl J Med. 2000;342:1500-7.

[6] Gomez R, Romero R, Nien JK, Medina L, Carstens M, Kim $\mathrm{YM}$, et al. Antibiotic administration to patients with preterm premature rupture of membranes does not eradicate intraamniotic infection. J Matern Fetal Neonatal Med. 2007;20: $167-73$.

[7] González-Luis G, Jordán García I, Rodríguez-Miguélez J, Botet Mussons F, Figueras Aloy J. Neonatal morbidity and mortality in very low birth weight infants according to exposure to chorioamnionitis. An Esp Pediatr. 2002;56:551-5.

[8] Hagberg H, Wennerholm UB, Sävman K. Sequelae of chorioamnionitis. Curr Opin Infect Dis. 2002;15:301-6.

[9] Herbst A, Nilsson C. Diagnosis of early preterm labour. Br J Obstet Gynaecol. 2006;113(Suppl 3):60-7.

[10] Holzman C, Lin X, Senagore P, Chung H. Histologic chorioamnionitis and preterm delivery. Am J Epidemiol. 2007; 177:786-94.

[11] Kallapur SG, Jobe AH. Contribution of inflammation to lung injury and development. Arch Dis Child Fetal Neonatal Ed. 2006;91:F132-5.

[12] Miyazaki K, Furuhashi M, Matsuo K, Minami K, Yoshida K, Kuno N, et al. Impact of subclinical chorioamnionitis on maternal and neonatal outcomes. Acta Obstet Gynecol Scand. 2007;86:191-7.

[13] Mu SC, Lin CH, Chen YL, Ma HJ, Lee JS, Lin MI, et al. Impact on neonatal outcome and anthropometric growth in very low birth weight infants with histological chorioamnionitis. J Formos Med Assoc. 2008;107:304-10.

[14] Newton ER. Preterm labor, preterm premature rupture of membranes, and chorioamnionitis. Clin Perinatol. 2005;32: $571-600$ 
[15] Oboro VO, Adekanle BA, Apantaku BD, Onadipe OA. Preterm pre-labour rupture of membranes: effect of chorioamnionitis on overall neonatal outcome. J Obstet Gynaecol. 2006;26:740-3.

[16] Ramsey PS, Lieman JM, Brumfield CG, Carlo W. Chorioamnionitis increases neonatal morbidity in pregnancies complicated by preterm premature rupture of membranes. Am J Obstet Gynecol. 2005;192:1162-6.

[17] Rocha G, Proença E, Quintas C, Rodrigues T, Guimarães H. Chorioamnionitis and neonatal morbidity. Acta Med Port. 2006;19:207-12.

[18] Romero R, Espinoza J, Gonçalves LF, Kusanovic JP, Friel L, Hassan S. The role of inflammation and infection in preterm birth. Semin Reprod Med. 2007;25:21-39.

[19] Soraisham AS, Singhal N, McMillan DD, Sauve RS, Lee SK. A multicenter study on the clinical outcome of chorioamnionitis in preterm infants. Am J Obstet Gynecol. 2009;200: 372.e1-6.
[20] Suppiej A, Franzoi M, Vedovato S, Marucco A, Chiarelli S, Zanardo V. Neurodevelopmental outcome in preterm histological chorioamnionitis. Early Hum Dev. 2009;85:187-9.

[21] Willoughby RE Jr, Nelson KB. Chorioamnionitis and brain injury. Clin Perinatol. 2002;29:603-21.

[22] Wu YW, Colford JM Jr. Chorioamnionitis as a risk factor for cerebral palsy: a meta-analysis. J Am Med Assoc. 2000;284: 1417-24.

[23] Yoon BH, Park CW, Chaiworapongsa T. Intrauterine infection and the development of cerebral palsy. Br J Obstet Gynaecol. 2003;110(Suppl 20):124-7.

The authors stated that there are no conflicts of interest regarding the publication of this article.

Received May 29, 2009. Revised August 27, 2009. Accepted October 7, 2009. Previously published online February 2, 2010. 